\title{
Observability of Market Daily Volatility
}

\author{
Filippo Petroni ${ }^{\mathrm{a}, *}$, Maurizio Serva ${ }^{\mathrm{b}}$ \\ ${ }^{a}$ Dipartimento di Scienze Economiche ed Aziendali, Università di Cagliari, Italy \\ ${ }^{b}$ Dipartimento di Ingegneria e Scienze dell'Informazione e Matematica, Università \\ dell'Aquila, Italy
}

\begin{abstract}
We study the price dynamics of 65 stocks from the Dow Jones Composite Average from 1973 until 2014. We show that it is possible to define a Daily Market Volatility $\sigma(t)$ which is directly observable from data. This quantity is usually indirectly defined by $r(t)=\sigma(t) \omega(t)$ where the $r(t)$ are the daily returns of the market index and the $\omega(t)$ are i.i.d. random variables with vanishing average and unitary variance. The relation $r(t)=\sigma(t) \omega(t)$ alone is unable to give an operative definition of the index volatility, which remains unobservable. On the contrary, we show that using the whole information available in the market, the index volatility can be operatively defined and detected.
\end{abstract}

Keywords: Market volatility; Absolute returns; Long-range auto-correlations; Cross-correlations.

\footnotetext{
* Corresponding author.

Email address: fpetroni@unica.it (Filippo Petroni)
} 
It is well know that stock market returns are uncorrelated on lags larger than a single day. This is an inescapable consequence of the efficiency of markets. On the contrary, absolute returns have memory for very long times, a phenomenon known as volatility clustering. These phenomena are very well documented in the literature and known as stylized facts [1, 2, 3, 4.

Furthermore, there is a large empirical evidence that volatility auto-correlations decay hyperbolically [5, 6, 7, 8, 9, and there is also a growing evidence that volatility signals have a multi-fractal nature $[10,11,12,13,14,15$.

Daily historical volatility is an unobservable variable and is usually measured by the absolute value of daily returns which are instead observable. This definition gives only an approximation of the real volatility $\sigma(t)$ which can be indirectly defined by $r(t)=\sigma(t) \omega(t)$ where $r(t)$ are the daily returns of the market index and the $\omega(t)$ are i.i.d. random variables with vanishing average and unitary variance. The relation $r(t)=\sigma(t) \omega(t)$ alone is unable to give an operative definition of the index volatility, which remains unobservable. We will show that using the whole information available in the market, the index volatility can be operatively defined and detected, i.e., we will define an observable volatility for a market index which exhibits all the statistical features expected for this variable.

The daily returns of single stock (say $\alpha$ ) are given by $r_{\alpha}(t)=\ln \left[S_{\alpha}(t) / S_{\alpha}(t-\right.$ $1)$ ] where $S_{\alpha}(t)$ is the closing price of stock $\alpha$ at day $t$. Then, if one wants to extract volatility from data one can consider that $r_{\alpha}(t)=\sigma_{\alpha}(t) \omega_{\alpha}(t)$ where the $\omega(t)$ have vanishing averages and unitary variance. Volatility $\sigma_{\alpha}(t)$ can be eventually extracted considering the high frequency (intra-day) continuous trading, but the problem remains highly unsolved because of the overnight contribution to the return $r_{\alpha}(t)$ for which there is not continuous trading. Therefore, the best measure of (historical) daily volatility simply remain the absolute returns $\left|r_{\alpha}(t)\right|$.

If the aim is to measure the global volatility of a market, we will show that things can be different. One can address the problem considering volatility of a proper representative index. Nevertheless, if one considers a price-weighted index (as Nikkei 225) the main contributions will be artificially given by those stocks with a larger price. The problem is circumvented if one considers a capitalization-weighted index (as Hang Seng) or an equally-weighted index (as $\mathrm{S} \& \mathrm{P} 500 \mathrm{EWI}$ ). The daily return $r(t)$ of this last index is simply the plain average of the returns of its components. i.e.

$$
r(t)=\frac{1}{N} \sum_{\alpha=1}^{N} r_{\alpha}(t)
$$

where $N$ is the number of stocks in the basket and $r_{\alpha}(t)=\ln \left(S_{\alpha}(t) / S_{\alpha}(t-1)\right)$ and $S_{\alpha}(t)$ is the daily closing price of the stock $\alpha$ at day $t$. For the other two types of index the difference is that the average is weighed by price or by capitalization.

Again, the underlying index daily volatility $\sigma(t)$ is not directly observable from daily returns but it is indirectly defined by $r(t)=\sigma(t) \omega(t)$. Because of 
market efficiency, it can be assumed that the $\omega(t)$ are independent identically distributed random variables with vanishing average and unitary variance. One could argue that $\sigma(t)$ is, indeed, observable from high frequency data, but, as already mentioned, the problem of the overnight contribution to the daily returns remains.

Therefore, since daily market volatility is not objectively given by the index return (only the product $\sigma(t) \omega(t)$ is observable), its distribution depends on the model chosen for $\omega(t)$. Gaussianity is often assumed as in ARCH-GARCH modeling (the leptokurticity of the distribution of returns is, in this case, entirely charged to volatility). Nevertheless, one can do other choices for the distribution of $\omega(t)$ as, for example, the uniform distribution (between $-\sqrt{3}$ and $\sqrt{3}$ in order that the variance is unitary).

In this paper we consider $N=65$ titles of Dow Jones from 1973 to 2014 so that $1 \leq t \leq T \simeq 10000$. Dow Jones as an index is not equally weighted but we can construct ourselves an EW Dow Jones index for which the daily returns $r(t)$ are simply the plain average of returns of its components as in definition (1).

The absolute returns are then given by

$$
|r(t)|=\sigma(t)|\omega(t)|=\frac{1}{N}\left|\sum_{\alpha=1}^{N} r_{\alpha}(t)\right|
$$

which are the absolute returns of the associated equally-weighted index (for price-weighted and capitalization-weighted indexes the only difference is that some weights must be introduced).

The core of this paper is the definition of the volatility as

$$
\sigma(t)=\frac{1}{\sqrt{3} N} \sum_{\alpha=1}^{N}\left|r_{\alpha}(t)\right|
$$

so that

$$
\omega(t)=\frac{r(t)}{\sigma(t)}
$$

where $r(t)$ and $\sigma(t)$ are defined in equation (1) and equation (3).

Most of the models assume that $\sigma(t)$ and $|\omega(t+\tau)|$ are uncorrelated for any value of the lag $\tau$ (negative, positive or vanishing) or they assume (as ARCHGARCH) a short range correlation (a correlation only for small values of $|\tau|$.) Moreover $|r(t)|$ and $\mid \omega(t+\tau)) \mid$ should be uncorrelated for any non vanishing $\tau$ as well as $|\omega(t)|$ and $|\omega(t+\tau)|$.

Therefore, the first step is to show that all these properties holds according to our definition of volatility. After having computed the correlations $C_{\omega, \sigma}=$ $C(\omega(t), \sigma(t+\tau)), C_{\sigma, \omega}=C(\sigma(t), \omega(t+\tau))$ and also $C_{\omega, \omega}, C_{\omega,|r|}$ and $C_{|r|, \omega}$, we have plotted them in Fig. 1. As it can be well appreciated the four above considered cross-correlations and the auto-correlation substantially vanish. This 
is better appreciated if compared with the volatility auto-correlation $C_{\sigma, \sigma}$ which is also plotted and which, on the contrary, exhibits a strong lag dependence and it is significantly positive for lags up to 250 working days.

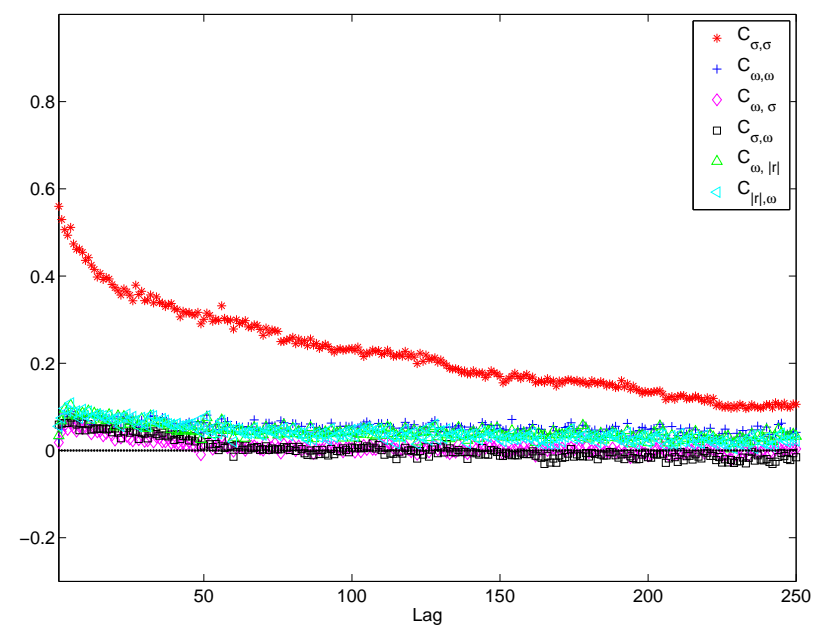

Figure 1: Auto-correlation of $\sigma,|\omega|$ and all the cross-correlations between $\sigma, \omega$ and $|r|$. It can be noticed that all except the auto-correlation of $\sigma$ are vanishing.

Once shown that the variables $\omega(t)$ are independent from each other, from the absolute returns (2) and from the volatilities (3), we need to show that they have vanishing expected value $(\langle\omega(t)\rangle=0)$ and unitary variance $\left(\left\langle\omega^{2}(t)\right\rangle=1\right)$. Indeed, we obtain a better result, in fact, the distribution of the $\omega(t)$ is uniform in the range $[-\sqrt{3}, \sqrt{3}]$ (which implies vanishing expected value and unitary variance but also implies $\langle|\omega(t)|\rangle=\sqrt{3} / 2)$. This result, which is our second step, can be appreciated in Fig. 2 where the empirical distribution or $|\omega|$, as defined by (4), is plotted.

We still have a third step, to complete our argument. Having assumed complete independence of the $\omega(t)$ from the volatilities one forcefully has that the auto-correlation $C_{|r|,|r|}$ only differs for a positive multiplicative constant $0<k<1$ from the auto-correlation $C_{\sigma, \sigma}$ at any time lag $\tau \geq 1$. In fact, the auto-correlation of the absolute returns is

$$
C_{|r|,|r|}(\tau)=\frac{\langle|r(t+\tau)||r(t)|\rangle-\langle|r(t)|\rangle^{2}}{\left\langle r^{2}(t)\right\rangle-\langle|r(t)|\rangle^{2}}
$$

then taking into account that $|r(t)|=\sigma(t)|\omega(t)|$ and assuming mutual independence between the $\sigma(t)$ and the $\omega(t)$, one has for any $\tau \geq 1$ :

$$
C_{|r|,|r|}(\tau)=k C_{\sigma, \sigma}(\tau)
$$




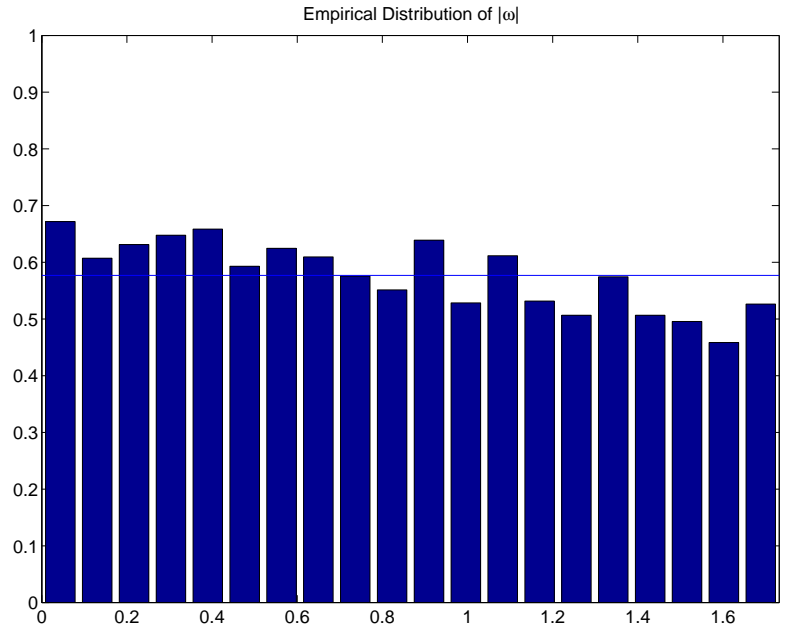

Figure 2: Uniform (almost) probability density of the $|\omega|$. One has $\left\langle\omega^{2}\right\rangle=0.924 \simeq 1$ ) and $\langle|\omega|\rangle=0.823 \simeq \sqrt{3} / 2=0.866$.

where $C_{\sigma, \sigma}(\tau)$ is the volatility auto-correlation

$$
C_{\sigma, \sigma}(\tau)=\frac{\langle\sigma(t+\tau) \sigma(t)\rangle-\langle\sigma(t)\rangle^{2}}{\left\langle\sigma^{2}(t)\right\rangle-\langle\sigma(t)\rangle^{2}}
$$

and $k$ is the constant

$$
k=\frac{\left\langle\sigma^{2}(t)\right\rangle-\langle\sigma(t)\rangle^{2}}{3\left\langle\sigma^{2}(t)\right\rangle / 4-\langle\sigma(t)\rangle^{2}}
$$

where we have used the uniform distribution value $\langle|\omega(t)|\rangle^{2} /\left\langle\omega^{2}(t)\right\rangle=3 / 4$. We compute from sample $\left\langle\sigma^{2}(t)\right\rangle=0.00008583$ and $\langle\sigma(t)\rangle=0.008388$ so that $k=1 / 2.85$.

Moreover, following the same steps, one can easily compute

$$
C_{|r|, \sigma}(\tau)=C_{\sigma,|r|}(\tau)=\sqrt{k} C_{\sigma, \sigma}(\tau)
$$

where $k$ is the same value (8) previously computed $(k \simeq 1 / 2.85)$ so that $(6)$ and (9) are very strict requirements. It turns out that both relations (6) and (9) hold, in fact, the four correlations, rescaled by $k$ (or by $\sqrt{k}$ ), are plotted in Fig. 3 where it can be appreciated that they are almost identical for all values of the $\operatorname{lag} \tau$.

Notice, that the coefficient $k$ is not a fitting parameter, but it is in dependently derived by market data. The fact that after rescaling the four correlations coincide ultimately confirms that it is correct to write $r(t)=\sigma(t) \omega(t)$ where $\omega(t)$ and $\sigma(t)$ are the mutually independent variables we have defined. 


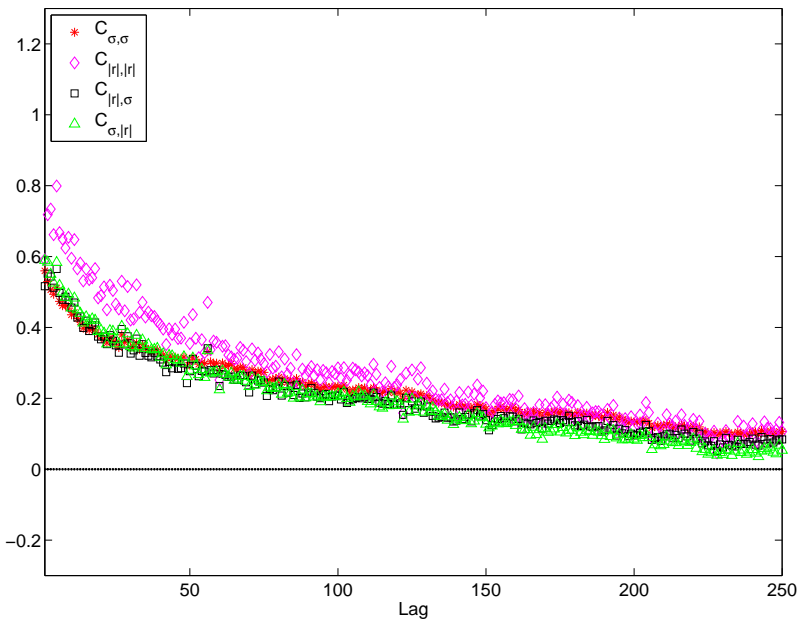

Figure 3: Auto-correlations of $\sigma$ and $|r|$ and their cross-correlations. It is evident that the four correlations are (almost) identical once rescaled.

In sum, we have defined the volatilities $\sigma(t)$ and, consequently, the variables $\omega(t)$ so that they (a) are mutually independent, (b) the $\omega(t)$ are also independent from absolute returns, (c) the $\omega(t)$ are i.i.d. uniformly distributed with vanishing expected value and unitary variance, (d) the auto-correlation of the volatility exhibits a strong lag dependence and it is significantly positive for lags up to 250 working days, (e) the correct scaling of cross-correlations and auto-correlations involving the $r(t)=\sigma(t) \omega(t)$ and the $\sigma(t)$ holds. Therefore, we have given a definition of daily market volatility which is observable and keep all the statistical features expected for this variable.

We can conclude by saying that while for a single stock it is impossible to extract the volatility from return using $r(t)=\sigma(t) \omega(t)$, we have found a simple way to do that for an index using all absolute returns of the single stocks entering in the index.

\section{References}

\section{References}

[1] Guillaume, D.M., Dacorogna, M.M., Dave, R.R., Müller, J.A., Olsen, R.B., Pictet, O.V., 1997. From the birds eye to the microscope: A survey of new stylized facts of the intra-daily foreign exchange markets, Finance \& Stochastic, 195.

[2] D'Amico, G., Petroni, F., 2012. Weighted-indexed semi-Markov models for modeling financial returns. Journal of Statistical Mechanics: Theory and Experiment, P07015. 
[3] D'Amico, G., Petroni, F., 2012. A semi-Markov model for price returns. Physica A Statistical Mechanics and its Applications, Vol. 391, 4867-4876.

[4] D'Amico, G., Petroni, F., 2011. A semi-Markov model with memory for price changes. Journal of Statistical Mechanics: Theory and Experiment P12009.

[5] Taylor, S., 1986. Modelling Financial Time Series. John Wiley \& Sons, New York.

[6] Ding, Z., Granger, C.W.J., Engle, R.F., 1993. A long memory property of stock market returns and a new model. Journal of Empirical Finance 1, 83106 .

[7] Baillie, R.T., Bollerslev, T., 1994. The long memory of the forward premium. Journal of International Money and Finance 13, 565572.

[8] Crato, N., de Lima, P., 1994. Long-range dependence in the conditional variance of stock returns. Economics Letters 45, 281-285.

[9] Pagan, A., 1996. The econometrics of financial markets. Journal of Empirical Finance 3, 15-102.

[10] Pasquini, M., Serva, M., 1999. Multiscale behaviour of volatility autocorrelations in a financial market. Economics Letters 65, 275-279.

[11] Pasquini, M., Serva, M., 2000. Clustering of volatility as a multiscale phenomenon. European Physical Journal B 16, 195-201.

[12] Pasquini, M., Serva, M., 1999. Multiscaling and clustering of volatility. Physica A 269, 140-147.

[13] Baviera, R., Pasquini, M., Serva, M., Vergni, D., Vulpiani, A., 2001. Correlations and multiaffinity in high frequency financial datasets. Physica A $300,551-557$.

[14] Wang, F., Yamasaki, K., Havlin, S., Stanley H.E., 2008. Indication of multiscaling in the volatility return intervals of stock markets. Physical Review E 77, 016109.

[15] Miccichè, S., 2013. Empirical relationship between stocks' crosscorrelation and stocks' volatility clustering. Journal of Statistical mechanics, P05015.

[16] Lillo, F., Mantegna, R.N., 2000. Variety and volatility in financial markets. Physical Review E 62, 6126-6134. 